\title{
Sporadic and Severe Lower Respiratory Illness- related Human Adenovirus Type 21 Infection in Southern China
}

\section{Wenkuan Liu}

Guangzhou Medical University

\section{Li Zhang}

Guangzhou Medical University

\section{Yong Cai}

Guangzhou Medical University

\section{Dehui Chen}

Guangzhou Medical University

\section{Shuyan Qiu}

Guangzhou Medical University

\section{Yanqun Wang}

Guangzhou Medical University

\section{Duo Xu}

Guangzhou Medical University

\section{Shujun Gu}

Guangzhou Medical University

\section{Xiao Li}

Guangzhou Medical University

\section{Jing Dai}

Guangzhou Medical University

\section{Qian Liu}

The First Affiliated Hospital of Guangdong Pharmaceutical University

\section{Xingui Tian}

Guangzhou Medical University

Rong Zhou ( $\sim$ zhourong@gird.cn )

Guangzhou Medical University https://orcid.org/0000-0001-7322-3561

\section{Research Article}

Keywords: human adenovirus type 21, severe lower respiratory illness, epidemiology, genome, cytopathogenicity 
Posted Date: September 16th, 2021

DOl: https://doi.org/10.21203/rs.3.rs-882659/v1

License: (c) (i) This work is licensed under a Creative Commons Attribution 4.0 International License. Read Full License 


\section{Abstract}

Background: Human adenovirus type 21 (HAdV-21) is an important member of HAdV species B, but our understanding of this type is limited.

Methods: We screened HAdV and 17 other common respiratory pathogens for 1,704 pediatric patients ( $\leq 14$ years old) hospitalized with acute respiratory illness in Guangzhou, China in 2019. HAdV-21 infections were further confirmed by molecular typing from HAdV-positive patients, and their clinical manifestations, genomes, infectivity and pathogenicity in vitro were analyzed.

Results: 151 of 1,704 cases (8.9\%) were positive for HAdV, making it the third most frequently detected pathogen. Two sporadic HAdV-21 infections were identified in June and September. Both HAdV-21positive patients presented with severe lower respiratory illness and had similar initial symptoms at onset of illness. The genome structure of HAdV-21 was found to be similar to that of other members of HAdV species B. The phylogenetic analysis showed that it was closely related to HAdV-B21 strain BB/201903 (MN686206) isolated in Bengbu, China in 2019, suggesting the possibility of the same source, and attention need to be paid to its prevention and control. In vitro, the infectivity and pathogenicity of HAdV21 were lower than the main epidemic types 7 and 3 . Plaques formed by HAdV-21, -7 , and -3 were significantly different in shape and size $(p<0.05)$, with plaques formed by HAdV-21 being the smallest and with poorly defined edges. There was no significant difference between the plaques of the HAdV-21 isolates $(p>0.05)$.

Conclusions: This study provides an important reference for the in-depth understanding of the epidemiology and pathogenicity of HAdV-21, and suggests the necessity of HAdV-21 research, prevention and control.

\section{Introduction}

Human adenoviruses (HAdVs) are non-enveloped, double-stranded DNA viruses of the family Adenoviridae. More than 100 genotypes of HAdVs have been identified, which are classified into seven species $(A-G)(1,2)$. HAdVs are associated with a broad spectrum of clinical diseases, such as acute respiratory illness (ARI), conjunctivitis, gastrointestinal infections, and obesity (2-5). Members of species $B$ are known to cause human diseases, with HAdV types $3,7,14$, and 55 being the most common causes of respiratory disease outbreaks (6).

HAdV-21 is a member of species B, and was first isolated in 1956 from a 1-year-old child with trachoma and conjunctivitis in Saudi Arabia (7). HAdV-21 was later found to be associated with a variety of diseases, including ARI (8-12). Severe pneumonia, myocarditis, flaccid paralysis, and even fatal infections in both pediatric and adult patients have been reported (13-16). The circulation of ARIassociated HAdV-21 has been reported among military recruits and civilians in several developed countries $(8,17-19)$, and caused nosocomial infections in lung transplant patients at a large tertiary care hospital (20). However, data on HAdV-21 are limited as HAdV-21 infections have rarely been reported in 
regions outside North America and Europe and are especially rare in China (21). To better understand the epidemiology of HAdV-21, infection data from different regions are of great importance.

In this study, we analyzed the distribution of respiratory pathogens in 1,704 pediatric patients with ARI in Guangzhou, China, in 2019. Two patients with HAdV-21 displaying severe lower respiratory illness (LRI) were identified. The clinical features of these infected patients were assessed, and the genomic characteristics and cyto-pathogenicity of HAdV-21 in vitro were analyzed.

\section{Materials And Methods}

\section{Respiratory sample collection}

Respiratory samples, including throat swabs, sputum, and bronchoalveolar lavage fluid, from pediatric patients ( $\leq 14$ years old) hospitalized with ARI were collected for routine screening of respiratory viruses Mycoplasma pneumoniae (MP) and Chlamydophila pneumoniae (CP) in accordance with established clinical protocols at the First Affiliated Hospital of Guangzhou Medical University between January and December 2019 (22). The samples were refrigerated at $2-8^{\circ} \mathrm{C}$ in viral transport medium, transported on ice to the State Key Laboratory of Respiratory Diseases, and analyzed immediately or stored at $-80^{\circ} \mathrm{C}$ before analysis, as previously described (23). The patients' clinical presentations were collected from the medical records.

\section{Screening for HAdV and common respiratory pathogens}

Respiratory pathogen screening was conducted to detect HAdV and 17 other common respiratory pathogens, including influenza A virus (infA), influenza B virus (infB), respiratory syncytial virus (RSV), parainfluenza virus types 1-4 (PIV1-4), human metapneumovirus (HMPV), human rhinovirus (HRV), enterovirus (EV), four types of coronaviruses (HCoV-229E, -OC43, -NL63, and -HKU1), human bocavirus $(\mathrm{HBoV}), \mathrm{MP}$, and $\mathrm{CP}$ simultaneously using TaqMan real-time quantitative polymerase chain reaction (qPCR), as previously reported (24).

\section{HAdV-21 identification}

HAdV-positive samples were subjected to further molecular typing for HAdV-3, -7, -14, -21, -55, -C5, and -E4 using TaqMan qPCR. The specific primers, which probed the hexon or fiber genes of the different HAdV types, are shown in Table S1. Probe qPCR Mix (TaKaRa, Dalian, China) was used according to the manufacturer's protocol. Clinical characteristics, treatments, and outcomes of the HAdV-21-positive patients were collected retrospectively.

\section{Cells, HAdV stocks, and HAdV-21-positive sample culture}

A549 cells were cultured in Dulbecco's minimum essential medium (DMEM) (Gibco) supplemented with $10 \%(\mathrm{v} / \mathrm{v})$ fetal bovine serum (FBS) and $100 \mathrm{U} / \mathrm{mL}$ penicillin-streptomycin (Gibco) at $37^{\circ} \mathrm{C}$ and $5 \%(\mathrm{v} / \mathrm{v})$ $\mathrm{CO}_{2}$. HAdV-21-positive samples were cultured in the $\mathrm{A} 549$ cells at $37^{\circ} \mathrm{C}$ and $5 \% \mathrm{CO}_{2}$ and maintained under standard conditions in DMEM supplemented with $2 \%(\mathrm{v} / \mathrm{v}) \mathrm{FBS}$ and $100 \mathrm{U} / \mathrm{mL}$ penicillin-streptomycin. 
Inoculated cells were monitored daily for the cytopathic effect (CPE) and were harvested at almost full CPE. HAdV-3-Guangzhou01 (accession no. DQ099432), HAdV-7-CQ1198 (accession no. JX625134), and HAdV-21 reference stain AV-1645 (ATCC, accession no. AY601633) were used simultaneously for analysis of the cyto-pathogenicity of the HAdV-21 isolates. HAdV-3-Guangzhou01 and HAdV-7-CQ1198, from the State Key Laboratory of Respiratory Diseases, were collected from patients with severe pneumonia in Guangzhou in 2005 and Chongqing in 2010, respectively. HAdV-21-AV-1645, which was first isolated in 1956 from a 1-year-old child with trachoma and conjunctivitis in Saudi Arabia (7), was kindly provided by Prof. Chenyang Li (Hecin Scientific, Guangzhou, China).

\section{HAdV-21 genome sequencing and annotation}

HAdV-21-positive samples were cultured and harvested. Viral genomic DNA was extracted using a TaKaRa Mini BEST Viral RNA/DNA Extraction Kit Ver.5.0 (TaKaRa) according to the manufacturer's instructions. Next-generation sequencing was conducted with a Illumina NovaSeq 6000 sequencer following a protocol from Synbio-Technologies (paired-end, $2 \times 150 \mathrm{bp}$ ). The complete genome of HAdV21 was assembled using CLC Genomics Workbench 11.0. The complete genomes of the HAdV-21 isolates were annotated based on the annotation of HAdV-21 strain BB/201903 (accession no. MN686206). Complete genome sequences were logged in the GenBank database.

\section{Phylogenetic analysis and HAdV sequences used}

Phylogenetic analysis was performed using Molecular Evolutionary Genetics Analysis (MEGA) version 5.05 (25). Phylogenetic trees were constructed by the Neighbor-joining ( $N J)$ method with 1,000 bootstrap replicates and default settings for all other parameters. HAdV sequences of the penton base, hexon, and fiber genes, and the genomes for phylogenetic analyses retrieved from GenBank are summarized in Table 1. 
Table 1

Sequences of adenovirus species A-G used for phylogenetic analysis

\begin{tabular}{|c|c|c|c|c|}
\hline Type & Strain & Country & Year isolated & GenBank accession number \\
\hline \multirow[t]{25}{*}{ HAdV-B21 } & GZ06109\# & China & 2019 & MW091531 \\
\hline & GZ09107"\# & China & 2019 & MW151243 \\
\hline & BB/201903 & China & 2019 & MN686206 \\
\hline & AV-1645 & Saudi Arabia & 1956 & AY601633 \\
\hline & OHT-006 & USA & 2016 & MF502426 \\
\hline & LRTI-1 & Germany & 2005 & KF577595 \\
\hline & LRTI-8 & Germany & 2016 & KY307858 \\
\hline & PEL0066 & USS & 2005 & KJ364587 \\
\hline & NHRC 64589 & USA & 2007 & KJ364582 \\
\hline & NHRC 71227 & USA & 2005 & KJ364584 \\
\hline & NHRC 52331 & USA & 2006 & KJ364581 \\
\hline & NHRC 44288 & USA & 2006 & KJ364576 \\
\hline & NHRC 32389 & USA & 2005 & KJ364573 \\
\hline & NHRC 91447 & USA & 2007 & KJ364590 \\
\hline & LRTI-3 & Germany & 2010 & KF577593 \\
\hline & LRTI-5 & Germany & 2012 & KF802425 \\
\hline & LRTI-5 & Germany & 2012 & KF577597 \\
\hline & LRTI-4 & Germany & 2012 & KF938575 \\
\hline & LRTI-6 & Germany & 2013 & KF577598 \\
\hline & LRTI-7 & Switzerland & 2013 & KY307857 \\
\hline & Sibu-97 & Malaysia & 1997 & KY307860 \\
\hline & VRDL T87-0342 & USA & 1987 & KJ364591 \\
\hline & LRTI-9 & Switzerland & 2016 & KY307859 \\
\hline & LRTI-2 & Germany & 2008 & KF802426 \\
\hline & CDC RU8176 & USA & 1978 & KJ364577 \\
\hline
\end{tabular}

\#The HAdV-B21 isolated in this study. 


\begin{tabular}{|c|c|c|c|c|}
\hline Type & Strain & Country & Year isolated & GenBank accession number \\
\hline & NHRC 10030 & USA & 1998 & KJ364586 \\
\hline & CDC V1375E & USA & 1984 & KJ364579 \\
\hline & NHRC 20007 & USA & 1998 & KJ364580 \\
\hline & NHRC 63218 & USA & 2006 & KJ364575 \\
\hline & NHRC 71139 & USA & 2004 & KJ364583 \\
\hline & NHRC 71252 & USA & 2005 & KJ364585 \\
\hline & NHRC 5 & USA & 1996 & KJ364578 \\
\hline & CDC V2148A & USA & 1988 & KJ364588 \\
\hline & VRDL T97-1745 & USA & 1997 & KJ364589 \\
\hline & NHRC 32493 & USA & 2005 & KJ364574 \\
\hline & VRDL T98-1269 & USA & 1998 & KJ364592 \\
\hline & GER & Germany & & KF528688 \\
\hline SAdV-21 & Bertha & USA & 1954 & AC_000010 \\
\hline HAdV-B3 & GB & USA & 1953 & AY599834 \\
\hline HAdV-B7 & Gomen & USA & 1952 & AY594255 \\
\hline HAdV-B11 & Slobitski & USA & 1956 & NC_011202 \\
\hline HAdV-B14 & de Wit & Netherlands & 1955 & AY803294 \\
\hline HAdV-B55 & BJ01 & China & 2011 & JX491639 \\
\hline HAdV-B34 & Compton & USA & 1972 & AY737797 \\
\hline HAdV-B35 & Holden & USA & 1973 & AY128640 \\
\hline HAdV-B16 & ch. 79 & USA & 1955 & AY601636 \\
\hline HAdV-B50 & Wan & USA & 1988 & AY737798 \\
\hline HAdV-B66 & AY128640 & Argentina & 1987 & JN860676 \\
\hline HAdV-B68 & $\operatorname{Arg} 827 / 04$ & Argentina & 2004 & JN860678 \\
\hline HAdV-A12 & Huie & USA & 1954 & AC_000005 \\
\hline HAdV-C1 & Adenoid 71 & USA & 1953 & AF534906 \\
\hline HAdV-D9 & Hicks & USA & 1954 & AJ854486 \\
\hline
\end{tabular}




\begin{tabular}{|lllll|}
\hline Type & Strain & Country & Year isolated & GenBank accession number \\
\hline HAdV-E4 & RI-67 & USA & 1952 & AY594253 \\
\hline HAdV-F40 & Dugan & Netherlands & 1979 & NC_001454 \\
HAdV-G52 & T03-2244 & USA & 2003 & DQ923122 \\
\hline \#The HAdV-B21 isolated in this study. & & \\
\hline
\end{tabular}

\section{Viral plaque formation assay}

A549 cells were seeded into 6-well culture plates and incubated overnight to form dense monolayers with more than $90 \%$ confluence. After removal of the growth media, the cultures were inoculated with $0.4 \mathrm{~mL}$ of 10 -fold serial dilutions of the viral stocks and incubated for $1 \mathrm{~h}$ at $37^{\circ} \mathrm{C}$ with rocking every $15 \mathrm{~min}$. The viral inocula were removed by aspiration and $3 \mathrm{~mL}$ DMEM-agarose mulch ( $2 \%$ SeaPlaque GTG-agarose [Lonza] mixed 1:1 with 2× DMEM medium containing 4\% FBS) was added to each well. The agarose was allowed to solidify at room temperature $\left(20-26^{\circ} \mathrm{C}\right)$. Plaque plates were incubated at $37^{\circ} \mathrm{C}$ and $5 \% \mathrm{CO}_{2}$ for a total of 13 days, with $1.5 \mathrm{~mL} /$ well of DMEM-agarose mulch supplementation after 4 and 8 days. The plates were stained with $2 \mathrm{~mL} /$ well $20 \%$ ethanol, $2 \%$ paraformaldehyde, and $1 \%$ crystal violet overnight at room temperature. The diameters of the plaques were measured with the assistance of the VisionWorks software package.

\section{Statistical analysis}

Statistical analysis was performed using SPSS 19.0 (SPSS Inc., Chicago, IL). Differences between groups were calculated using the $t$-test and Mann-Whitney $U$ test. A p-value $<0.05$ (two-tailed) was considered statistically significant.

\section{Results}

\section{Overall respiratory pathogen infection}

Samples from 1,704 pediatric patients hospitalized with ARI were collected, with 845 (49.6\%) patients infected with one or more of the pathogens of interest. Of these, 151 patients (8.9\%) were identified with HAdV infections, which was the third most frequently detected pathogen in this study (Fig. 1).

\section{HAdV-21 identification}

Two of the 151 (1.3\%) HAdV-positive patients, GZ06109 and GZ09107, were diagnosed with HAdV-21 in June and September 2019. In the other 149 patients, HAdV-3 (47.0\%, 71/151), HAdV-7 (46.4\%, 70/151), HAdV-4 (4.0\%, 6/151), and HAdV-55 (1.3\%, 2/151) were detected, while HAdV-5 and HAdV-14 were not.

Clinical characteristics of the HAdV-21-positive patients 
Data on the clinical characteristics, treatments, and outcomes of the two HAdV-21-positive patients were collected (Table 2). Both patients had similar initial symptoms of fatigue at the onset of the disease, and both were diagnosed with severe LRI by radiologic testing. Patient GZ06109 also had sepsis, although no microbes were found by blood culture. The indexes of white cell count, procalcitonin (PCT), C-reactive protein (CRP), aspartate aminotransferase (AST), and D-dimer exceeded the upper limits of the normal ranges for both patients. Levels of creatine kinase (CK) and lactate dehydrogenase (LDH) were abnormal and differed between the two patients. While the patients had similar disease durations, patient GZ09107, who had severe pneumonia in both lungs, was hospitalized for longer (9 days) than patient GZ06109 (5 days; Table 2). 
Table 2

Clinical characteristics, treatments, and outcomes of the two patients infected with HAdV-21

Characteristic

HAdV-21-positive patient

GZ06109

GZ09107

Clinical characteristic

Gender

Male

Female

Age, year

4.6

1

Existing chronic

Rhinitis

Negative

disease

Initial symptom

Repeated coughing and fever

Repeated coughing and fever

The highest

40.5

40.2

temperature, ${ }^{\circ} \mathrm{C}$

Sputum production

Yes

Yes

Shortness of breath

No

Yes

Fatigue

Yes

Yes

Radiologic findings

Pneumonia

Pneumonia on both lungs

\section{Laboratory findings}

The blood oxygen

99

$88-90$

saturation under

inhalation, \%

Bacteria or fungus

culture

Negative

Negative

White-cell count, $\times 10^{9} / \mathrm{L}$

15.3, $\uparrow$

$10.3, \uparrow$

Lymphocyte count,

3.6

3.4

$\times 10^{9} / \mathrm{L}$

Platelet count, $\times 10^{9} / \mathrm{L}$

342

332

Hemoglobin, g/L

101

104

Normal index range of test items: white cell count, $4-10 \times 10^{9} / \mathrm{L}$; lymphocyte count, $0.9-5.2 \times 10^{9} / \mathrm{L}$; platelet count, $100-400 \times 10^{9} / \mathrm{L}$; hemoglobin, $120-150 \mathrm{~g} / \mathrm{L}$; procalcitonin, $0-0.05 \mathrm{ng} / \mathrm{mL}$; alanine aminotransferase, 5-40 U/L; C-reactive protein, 0-0.6 mg/dL; aspartate aminotransferase, 5-40 U/L;

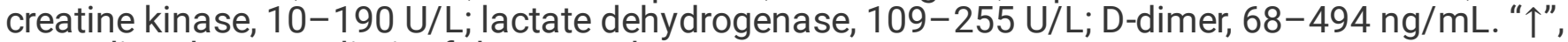
exceeding the upper limit of the normal range. 


\begin{tabular}{|c|c|c|}
\hline \multirow[t]{2}{*}{ Characteristic } & \multicolumn{2}{|l|}{ HAdV-21-positive patient } \\
\hline & GZ06109 & GZ09107 \\
\hline Procalcitonin, ng/mL & $1.47, \uparrow$ & $0.2, \uparrow$ \\
\hline $\begin{array}{l}\text { Alanine } \\
\text { aminotransferase, } \mathrm{U} / \mathrm{L}\end{array}$ & 15 & 12 \\
\hline $\begin{array}{l}\text { C-reactive protein, } \\
\mathrm{mg} / \mathrm{dL}\end{array}$ & $11.57, \uparrow$ & $1.02 \uparrow$ \\
\hline $\begin{array}{l}\text { Aspartate } \\
\text { aminotransferase, U/L }\end{array}$ & $51.1, \uparrow$ & $53, \uparrow$ \\
\hline Creatine kinase, $\mathrm{U} / \mathrm{L}$ & $1152, \uparrow$ & 86 \\
\hline $\begin{array}{l}\text { Lactate } \\
\text { dehydrogenase, U/L }\end{array}$ & 215 & $642, \uparrow$ \\
\hline D-dimer, ng/mL & $509, \uparrow$ & $1268, \uparrow$ \\
\hline \multicolumn{3}{|l|}{$\begin{array}{l}\text { Diagnosis of } \\
\text { physician }\end{array}$} \\
\hline & Pneumonia and sepsis & Severe pneumonia \\
\hline \multicolumn{3}{|l|}{ Treatments } \\
\hline $\begin{array}{l}\text { Symptomatic } \\
\text { treatment }\end{array}$ & $\begin{array}{l}\text { Anti-infection, anti-inflammation, } \\
\text { intravenous fluid therapy, atomization } \\
\text { inhalation treatment }\end{array}$ & $\begin{array}{l}\text { Anti-infection, anti- } \\
\text { inflammation, intravenous } \\
\text { fluid therapy }\end{array}$ \\
\hline $\begin{array}{l}\text { Intravenous immune } \\
\text { globulin }\end{array}$ & No & Yes \\
\hline $\begin{array}{l}\text { Mechanical } \\
\text { ventilation }\end{array}$ & No & Yes \\
\hline \multicolumn{3}{|l|}{$\begin{array}{l}\text { Clinical outcomes- } \\
\text { recovery duration }\end{array}$} \\
\hline $\begin{array}{l}\text { Total disease } \\
\text { duration, day }\end{array}$ & 20 & 19 \\
\hline $\begin{array}{l}\text { Length of hospital } \\
\text { stay, day }\end{array}$ & 5 & 9 \\
\hline \multicolumn{3}{|c|}{$\begin{array}{l}\text { Normal index range of test items: white cell count, } 4-10 \times 10^{9} / \mathrm{L} \text {; lymphocyte count, } 0.9-5.2 \times 10^{9} / \mathrm{L} \text {; } \\
\text { platelet count, } 100-400 \times 10^{9} / \mathrm{L} \text {; hemoglobin, } 120-150 \mathrm{~g} / \mathrm{L} ; \text { procalcitonin, } 0-0.05 \mathrm{ng} / \mathrm{mL} \text {; alanine } \\
\text { aminotransferase, } 5-40 \mathrm{U} / \mathrm{L} ; \mathrm{C} \text {-reactive protein, } 0-0.6 \mathrm{mg} / \mathrm{dL} ; \text { aspartate aminotransferase, } 5-40 \mathrm{U} / \mathrm{L} \\
\text { creatine kinase, } 10-190 \mathrm{U} / \mathrm{L} \text {; lactate dehydrogenase, } 109-255 \mathrm{U} / \mathrm{L} \text {; D-dimer, } 68-494 \mathrm{ng} / \mathrm{mL} \text {. " }{ }^{\prime \prime},\end{array}$} \\
\hline
\end{tabular}

\section{Genome features of HAdV-21 isolates}


HAdV-21 isolates GZ06109 and GZ09107 were sequenced, annotated, and uploaded to the GenBank database with accession numbers MW091531 and MW151243, respectively. The identified genomes were 35,362 and 35,365 bp in length for GZ06109 and GZ09107, respectively, and had similar genomic organization and transcription maps (shown for strain GZ09107 in Fig. 2).

\section{Phylogenetic features of the HAdV-21 isolates}

Phylogenetic analysis of the genomes and three structural protein sequences showed that HAdV-B21 members are closely related to each other, forming a clade (Fig. 3). The percent identities of the genomes and three capsid protein genes of the two HAdV-21 isolates identified here were compared with the most closely related strain, HAdV-21-BB/201903 (98.82-100\%), and the first strain to be isolated, HAdV-21-AV1645 (98.38-99.47\%; Table 3). Compared with HAdV-21-BB/201903, GZ06109 contained one insertion mutation in the penton base (GCG, alanine) and two synonymous mutations in the non-coding region. No non-synonymous mutations were found in strain GZ09107 (Fig. 4).

Table 3

Percent identities of genomes and capsid protein sequences of the isolated strains GZ06109 and GZ09107 with representative HAdV-21 strains

\begin{tabular}{|llllllllll|}
\hline Nucleic acid & GZ06109 & \multicolumn{7}{c|}{ GZ07109 } \\
\cline { 2 - 9 } & Genome & Hexon & $\begin{array}{l}\text { Penton } \\
\text { base }\end{array}$ & Fiber & Genome & Hexon & $\begin{array}{l}\text { Penton } \\
\text { base }\end{array}$ & Fiber \\
\hline BB/201903 & 99.97 & 100 & 98.82 & 100 & 99.97 & 100 & 100 & 100 \\
\hline AV-1645 & 98.86 & 99.16 & 99.28 & 99.38 & 98.86 & 99.16 & 99.47 & 99.38 \\
\hline a The most closely related strain, HAdV-21a/MN686206/CHN/BB/201903/2019; & \\
\hline bThe first HAdV-21 strain to be isolated, HAdV-B21/AY601633/Saudi Arabia/AV-1645/1956. \\
\hline
\end{tabular}

\section{Cyto-pathogenicity of the HAdV-21 isolates}

Cyto-pathogenicity analyses of HAdV-21 strains GZ06109, GZ09107, AV-1645, HAdV-3-Guangzhou01, and HAdV-7-CQ1198 were performed using the plaque formation assay (Fig. 5). Plaques formed by HAdV-7-CQ1198 and HAdV-3-Guangzhou01 were bigger and had clearer boundaries than those from the HAdV-21 strains ( $p<0.001$; Fig. 5A). Plaque sizes were significantly different among the three HAdV types $(p<0.05)$, but not among the three HAdV-21 strains (Fig. 5B).

\section{Discussion}

Among the more than 100 types and seven species (A-G) of HAdV, species B is of particular importance in ARI (http://hadvwg.gmu.edu/). Although HAdV-21 is an important member of HAdV species B, our understanding of this type is inadequate. From 2017 to 2019, HAdV strains were prevalent in China, 
causing outbreaks of severe pneumonia in children (http://www.chinacdc.cn/) (26). In this study, we analyzed the epidemiologic characteristics, genome features, and cyto-pathogenicity of HAdV-21

Of the 1,704 participants in this study, $8.9 \%$ of the patients were infected with HAdV, making it the third most prevalent pathogen detected (Fig. 1). This positive rate was higher than that reported for previous years in this region $(5 \%, 213 / 4242)(22)$. The main HAdV types detected were type $7(46.4 \%)$ and 3 (47.0\%), which is largely consistent with previous reports $(22,26,27)$. Two sporadic cases of HAdV-21, GZ06109 and GZ09107, were identified in June and September. The low prevalence of HAdV-21 may signify low immunity against this type in the general population, increasing its potential to cause an epidemic.

Both HAdV-21-positive patients presented with severe LRI (Table 2), which highlights the need for increased awareness of HAdV-21 infections. The HAdV-21 infections had similar initial symptoms at onset of illness, and multiple indexes exceeded the normal ranges, such as white cell count, PCT, CRP, AST, and D-Dimer. These factors may help physicians judge and screen for this pathogen (Table 2), although definitive diagnosis will require laboratory screening as many respiratory viruses have similar manifestations. Because there are no specific treatments against adenoviruses, only symptomatic treatment can be used at present. Previous studies have shown that HAdV-21 can cause nosocomial infections in immune-compromised patients (20), highlighting the importance of prevention and control of this type.

To improve our understanding of HAdV-21, we cultured the two HAdV-21 isolates, GZ06109 and GZ09107. Sequencing and annotation of their genomes revealed similar structures to other members of HAdV species B (28) (Fig. 2). By comparing and analyzing the genome sequences of HAdV-21 and other HAdV species (Table 1), we found that the HAdV-21 genome is comparatively stable and constitutes a clade (29) (Fig. 3). The two HAdV-21 strains in this study had highest genome identity (99.97\%) with strain HAdV-21-BB/201903 (accession no. MN686206), which was isolated in Bengbu, China in 2019, and 98.86\% similarity with the first strain to be isolated, HAdV-21-AV-1645 (Table 3). In terms of the main structural protein genes, GZ09107 had 100\% similarity to the Bengbu strain, with only two synonymous mutations ( $T$ antigen and 20k protein) and two non-coding region mutations (Fig. 4). Compared with the Bengbu strain, GZ06109 had 100\% sequence identity for the fiber and hexon genes (Table 3) and an alanine insertion in the penton base (Fig. 4). This suggests that HAdV-21 isolates prevalent in China have a high degree of kinship and are from the same source, although there is insufficient data to identify the potential source.

To analyze the pathogenicity of HAdV-21, plaque formation assays with the two HAdV-21 isolates in this study, reference HAdV-21-AV-1645, and severe pneumonia-related HAdV-3-Guangzhou01 and HAdV-7CQ1198 were conducted (Fig. 5). Although the plaques formed by the three HAdV-21 strains showed similar characteristics (Fig. 5A) and were similar in size ( $p>0.05$; Fig. 5B), they were significantly smaller $(p<0.001)$ than plaques from HAdV-7 and HAdV-3, and had poorly defined edges. It was also found that plaques formed by HAdV-7 were significantly larger than those of HAdV-3 $(p<0.05)$. These plaque 
features indicate that HAdV-21 < HAdV-3 < HAdV-7 with regard to virulence and infectivity. Compared with clinical research reports, the prevalence and pathogenicity of HAdV-7 and HAdV-3 are largely consistent with this result (30). There are too few reports on HAdV-21 to determine its overall pathogenic characteristics; thus, more research on this type is urgently needed.

The main limitation of this study is that selection bias may have occurred, because the sample comes from one hospital, and there is a lack of samples from outpatient clinics and healthy people. This may lead to deviations in the understanding of HAdV-21 infection, especially the epidemiological characteristics.

\section{Conclusions}

In this study, we investigated HAdV-21 infections in Guangzhou, China, and identified two patients infected with severe LRI-related HAdV-21 strains. Genomic analysis showed that the Chinese isolates of HAdV-21 showed a high degree of similarity, suggesting that attention should be paid to its prevention and control. Analyses of the clinical characteristics, genome structures, and cyto-pathogenicity of these strains revealed important information that will contribute to a deeper understanding of HAdV-21.

\section{Abbreviations}

HAdV: Human adenovirus

ARI: acute respiratory illness

LRI: lower respiratory illness

infA: influenza A virus

infB: influenza B virus

RSV: respiratory syncytial virus

PIV: parainfluenza virus

HMPV: human metapneumovirus

HRV: human rhinovirus

EV: enterovirus

HCoV: human coronaviruses

HBoV: human bocavirus

MP: Mycoplasma pneumoniae 
CP: Chlamydophila pneumoniae

qPCR: quantitative polymerase chain reaction

DMEM: Dulbecco's minimum essential medium

FBS: fetal bovine serum

CPE: cytopathic effect

MEGA: Molecular Evolutionary Genetics Analysis

$\mathrm{NJ}$ : Neighbor-joining

PCT: procalcitonin

CRP: C-reactive protein

AST: aspartate aminotransferase

CK: creatine kinase

LDH: lactate dehydrogenase

\section{Declarations}

\section{Ethics approval and consent to participate}

The First Affiliated Hospital of Guangzhou Medical University Ethics Committee approved the involvement of human subjects in this study. Next of kin, caretakers, or guardians gave signed informed consent for participation in the study on behalf of the minors/children.

\section{Consent for publication}

Not applicable.

\section{Availability of data and materials}

The datasets used and/or analysed during the current study are available from the corresponding author on reasonable request.

\section{Competing interests}

The authors declare that they have no competing interests.

\section{Funding}


This study was supported by Guangzhou Science and Technology Program-Zhongnanshan Medical Foundation of Guangdong Province (202102010359-ZNSA-2020003); Emergency Key Program of Guangzhou Laboratory (EKPG21-13); Special Project for COVID-19 Prevention and Control of Zhongnanshan Medical Foundation of Guangdong Province (ZNSA-2020012); National Natural Science Foundation of China (81970003, 31900877); and the Natural Science Foundation of Guangdong Province of China (2018A030310401). The funders had no input in the study design, data collection, or interpretation, or the decision to submit the work for publication.

\section{Authors' contributions}

Conceptualization: WKL, QL, XGT, and RZ; Methodology: WKL, LZ, YC, and RZ; Formal analysis: WKL, LZ, YC, QL, XGT, and RZ; Investigation: WKL, LZ, YC, YSQ, YQW, DX, SJG, XL, and JD; Resources: DHC, QL; Writing - Original Draft Preparation: WKL, LZ, YC, and QL; Writing - Review \& Editing: XGT and RZ; Funding Acquisition: WKL, RZ, and QL. All authors have read and agreed on the final manuscript.

\section{Acknowledgments}

We thank the study volunteers for their participation, and Prof. Chenyang Li for generously providing strain AV-1645. We also thank Yinghua Zhou and Jing Ma for their technical assistance.

\section{References}

1. Ismail AM, Lee JS, Lee JY, Singh G, Dyer DW, Seto D, et al. Adenoviromics: Mining the Human Adenovirus Species D Genome. Front Microbiol. 2018;9:2178.

2. Ji T, Li L, Li W, Zheng X, Ye X, Chen H, et al. Emergence and characterization of a putative novel human adenovirus recombinant HAdV-C104 causing pneumonia in Southern China. Virus Evol. 2021;7(1):veab018.

3. Radke JR, Cook JL. Human adenovirus infections: update and consideration of mechanisms of viral persistence. Curr Opin Infect Dis. 2018;31(3):251-6.

4. Sandkovsky U, Vargas L, Florescu DF. Adenovirus: current epidemiology and emerging approaches to prevention and treatment. Curr Infect Dis Rep. 2014;16(8):416.

5. Chen SY, Liu W, Xu Y, Qiu S, Chen Y, Tian X, et al. Epidemiology and Genetic Variabilities of Human Adenovirus Type 55 Reveal Relative Genome Stability Across Time and Geographic Space in China. Front Microbiol. 2020;11:606195.

6. Prusinkiewicz MA, Mymryk JS. Metabolic Reprogramming of the Host Cell by Human Adenovirus Infection. Viruses. 2019;11(2).

7. Bell SD, Jr., Mc CD, Murray ES, Chang RS, Snyder JC. Adenoviruses isolated from Saudi Arabia. I. Epidemiologic features. Am J Trop Med Hyg. 1959;8(4):492-500.

8. James AG, Lang WR, Liang AY, Mackay RJ, Morris MC, Newman JN, et al. Adenovirus type 21 bronchopneumonia in infants and young children. J Pediatr. 1979;95(4):530-3. 
9. Wright J, Couchonnal G, Hodges GR. Adenovirus type 21 infection. Occurrence with pneumonia, rhabdomyolysis, and myoglobinuria in an adult. JAMA. 1979;241(22):2420-1.

10. Darougar S, Pearce R, Gibson JA, McSwiggan DA. Adenovirus type 21 keratoconjunctivitis. The British journal of ophthalmology. 1978;62(12):836-7.

11. Becroft DM. Bronchiolitis obliterans, bronchiectasis, and other sequelae of adenovirus type 21 infection in young children. J Clin Pathol. 1971;24(1):72-82.

12. Lee CS, Lee AY, Akileswaran L, Stroman D, Najafi-Tagol K, Kleiboeker S, et al. Determinants of Outcomes of Adenoviral Keratoconjunctivitis. Ophthalmology. 2018;125(9):1344-53.

13. Lang WR, Howden CW, Laws J, Burton JF. Bronchopneumonia with serious sequelae in children with evidence of adenovirus type 21 infection. Br Med J. 1969;1(5636):73-9.

14. Hage E, Huzly D, Ganzenmueller T, Beck R, Schulz TF, Heim A. A human adenovirus species B subtype 21a associated with severe pneumonia. J Infect. 2014;69(5):490-9.

15. Henson D, Mufson MA. Myocarditis and pneumonitis with type 21 adenovirus infection. Association with fatal myocarditis and pneumonitis. Am J Dis Child. 1971;121(4):334-6.

16. Ooi MH, Wong SC, Clear D, Perera D, Krishnan S, Preston T, et al. Adenovirus type 21-associated acute flaccid paralysis during an outbreak of hand-foot-and-mouth disease in Sarawak, Malaysia. Clin Infect Dis. 2003;36(5):550-9.

17. Pereira MS. Occurrence of Adenovirus Type 21 in Great Britain. Br Med J. 1963;1(5332):728-9.

18. van der Veen J, Oei KG, Abarbanel MF. Patterns of infections with adenovirus types 4, 7 and 21 in military recruits during a 9-year survey. J Hyg (Lond). 1969;67(2):255-68.

19. van der Avoort HG, Adrian T, Wigand R, Wermenbol AG, Zomerdijk TP, de Jong JC. Molecular epidemiology of adenovirus type 21 in the Netherlands and the Federal Republic of Germany from 1960 to 1985. J Clin Microbiol. 1986;24(6):1084-8.

20. Philo SE, Anderson BD, Costa SF, Henshaw N, Lewis SS, Reynolds JM, et al. Adenovirus Type 21 Outbreak Among Lung Transplant Patients at a Large Tertiary Care Hospital. Open Forum Infect Dis. 2018;5(8):ofy188.

21. Deng J, Qian Y, Zhao LQ, Zhu RN, Wang F, Sun Y. [Identification and typing for adenovirus by multiplex nest-PCR]. Zhonghua Liu Xing Bing Xue Za Zhi. 2007;28(8):781-4.

22. Liu WK, Liu Q, Chen de H, Liang HX, Chen XK, Chen MX, et al. Epidemiology of acute respiratory infections in children in guangzhou: a three-year study. PLoS One. 2014;9(5):e96674.

23. Liu WK, Chen DH, Liu Q, Liang HX, Yang ZF, Qin S, et al. Detection of human bocavirus from children and adults with acute respiratory tract illness in Guangzhou, southern China. BMC Infect Dis. 2011;11:345.

24. Liu WK, Liu Q, Chen DH, Liang HX, Chen XK, Huang WB, et al. Epidemiology and clinical presentation of the four human parainfluenza virus types. BMC Infect Dis. 2013;13:28.

25. Tamura K, Peterson D, Peterson N, Stecher G, Nei M, Kumar S. MEGA5: molecular evolutionary genetics analysis using maximum likelihood, evolutionary distance, and maximum parsimony 
methods. Mol Biol Evol. 2011;28(10):2731-9.

26. Duan Y, Li C, Deng L, An S, Zhu Y, Wang W, et al. Genetic Analysis of Human Adenovirus Type 7 Strains Circulating in Different Parts of China. Virol Sin. 2021.

27. Lin MR, Yang SL, Gong YN, Kuo CC, Chiu CH, Chen CJ, et al. Clinical and molecular features of adenovirus type 2, 3, and 7 infections in children in an outbreak in Taiwan, 2011. Clin Microbiol Infect. 2017;23(2):110-6.

28. Cheng Z, Yan Y, Jing S, Li WG, Chen WW, Zhang J, et al. Comparative Genomic Analysis of Reemergent Human Adenovirus Type 55 Pathogens Associated With Adult Severe Community-Acquired Pneumonia Reveals Conserved Genomes and Capsid Proteins. Front Microbiol. 2018;9:1180.

29. Kajon AE, Hang J, Hawksworth A, Metzgar D, Hage E, Hansen CJ, et al. Molecular Epidemiology of Adenovirus Type 21 Respiratory Strains Isolated From US Military Trainees (1996-2014). J Infect Dis. 2015;212(6):871-80.

30. Fu Y, Tang Z, Ye Z, Mo S, Tian X, Ni K, et al. Human adenovirus type 7 infection causes a more severe disease than type 3. BMC Infect Dis. 2019;19(1):36.

\section{Figures}




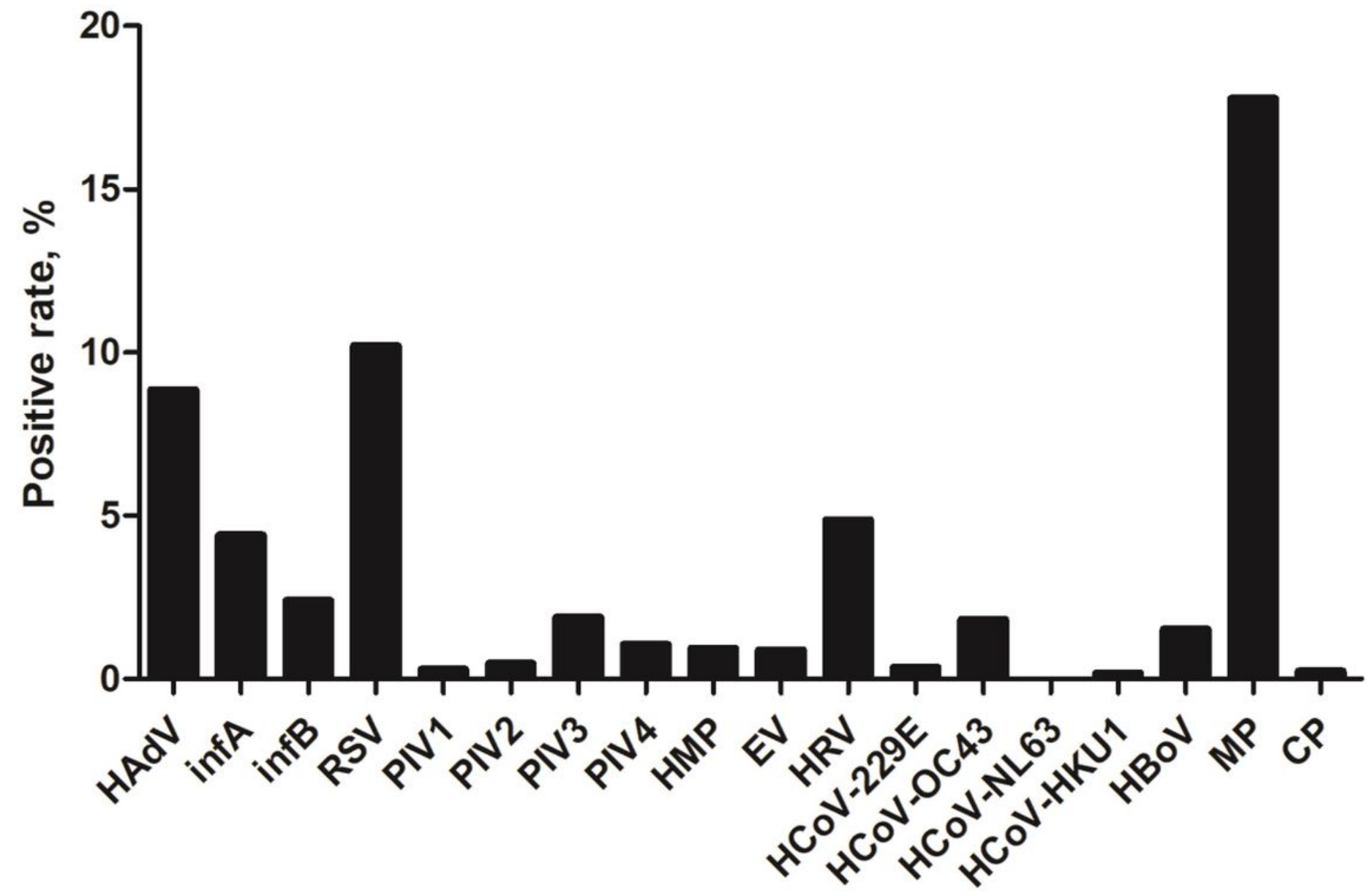

Figure 1

Distribution of respiratory pathogens in 1,704 children hospitalized with acute respiratory illness in Guangzhou, China in 2019

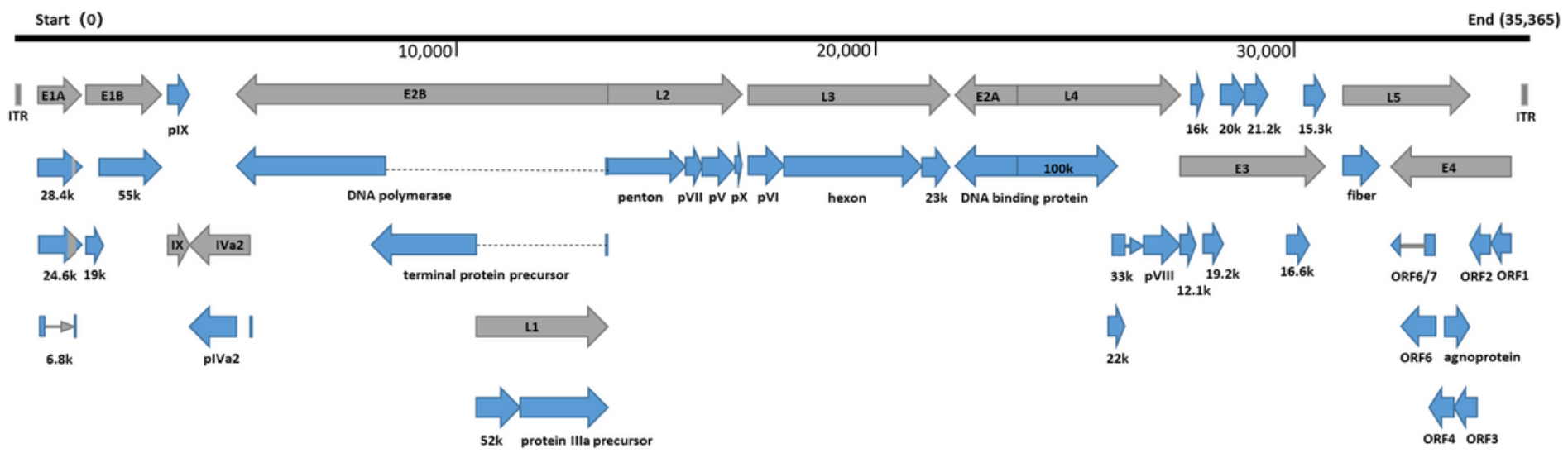

Figure 2 
Transcriptional map and genome organization of HAdV-21 strain GZ09107 The genome is indicated by the black horizontal line marked at 10,000 bp intervals. The transcription units are designated by gray arrows, while blue arrows designate coding regions. Arrows reflect the transcriptional orientation of the coding transcripts.
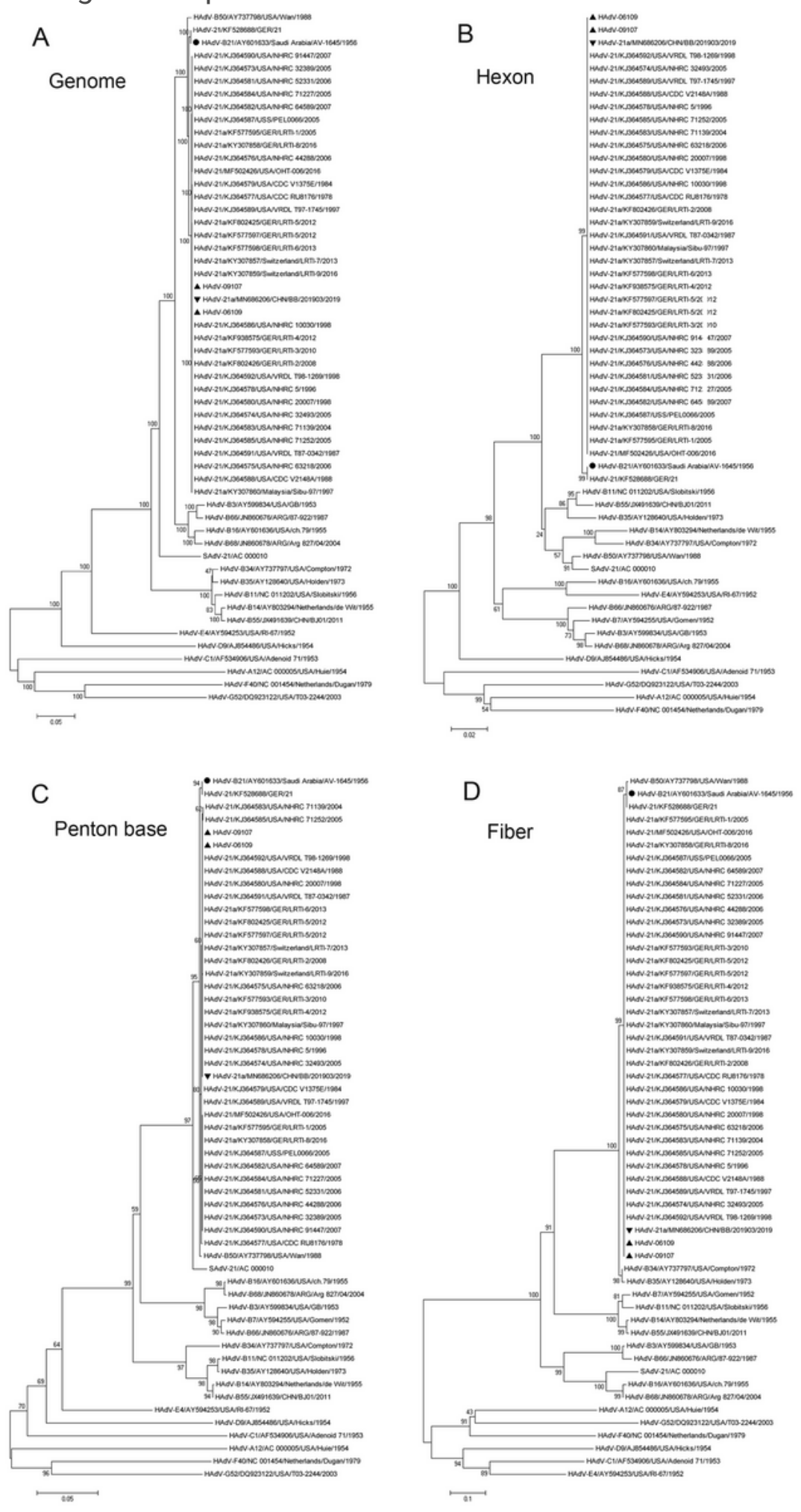

Figure 3 
Phylogenetic analysis of HAdV-21 strains GZ06109 and GZ09107 The nucleotide sequences of the whole genome (A), hexon (B), penton base (C), and fiber (D) genes of the HAdV strains were analyzed for their phylogenetic relationships using the Neighbor-Joining method with 1,000 bootstrap replicates implemented in the MEGA 5.0 software package. For reference, taxon names include the genome type, corresponding GenBank accession number, country of isolation, strain name, and year of isolation. The two HAdV-21 strains isolated in this study are marked with " $\boldsymbol{\Delta}^{\text {"; " }}$ " $\boldsymbol{\nabla}$ ", strain isolated from Bangbu (BB/201903), China in 2019; "O", reference standard HAdV-21 isolated in Saudi Arabia in 1945.

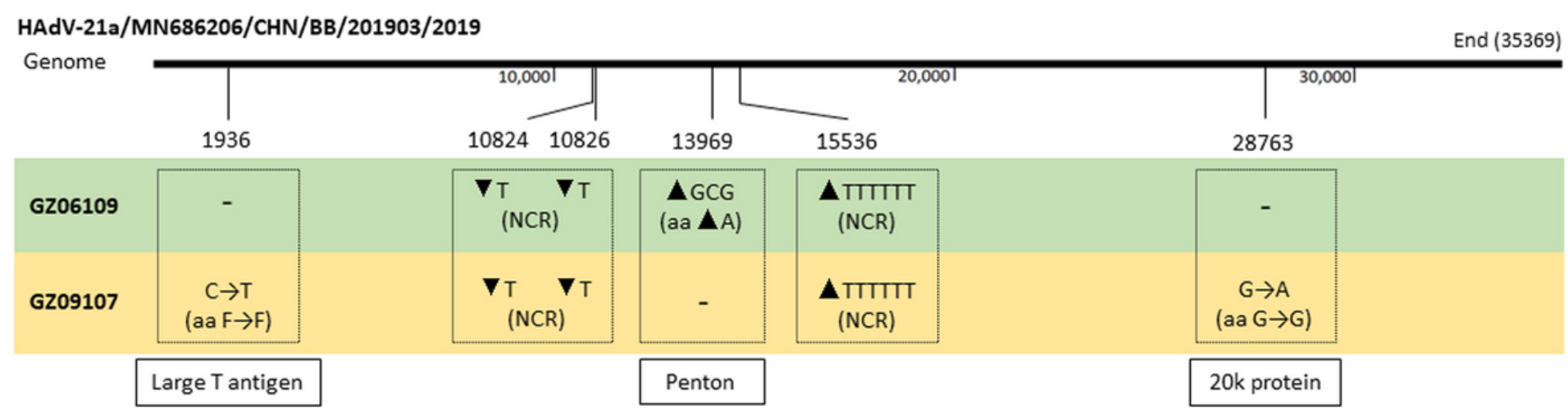

Figure 4

Comparative genomics analysis of the two HAdV-21 isolates and reference strain BB/201903 Nucleic acid and amino acid sequence changes are noted, along with their genome locations and coding consequences. The reference genome of strain HAdV-21 BB/201903 is indicated by the black horizontal line marked at 10,000 bp intervals. NCR, non-coding region; $\boldsymbol{\nabla}$, insertion; $\boldsymbol{\Lambda}$, deletion; - , no change.

A

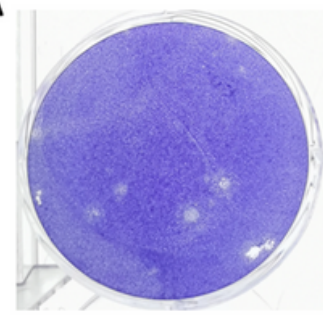

HAdV-21-AV-1645

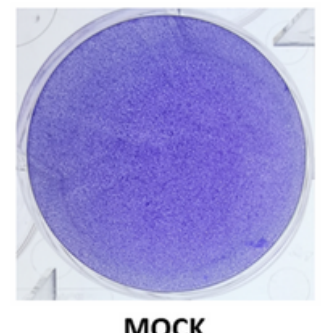

MOCK

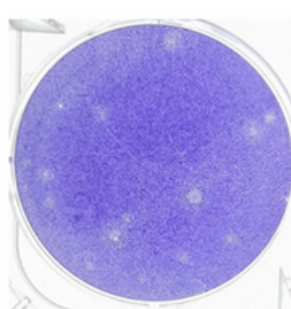

HAdV-21-GZ06109

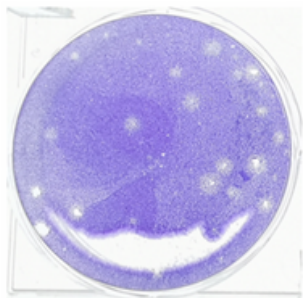

HAdV-3-Guangzhou01

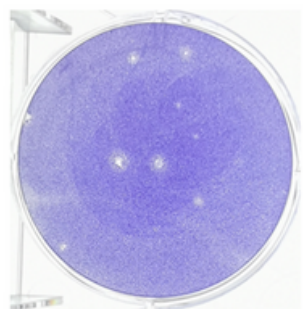

HAdV-21-GZ09107

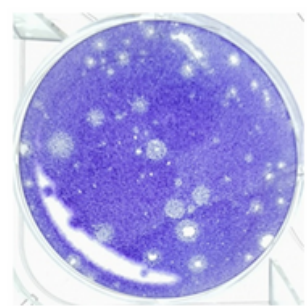

HAdV-7-CQ1198

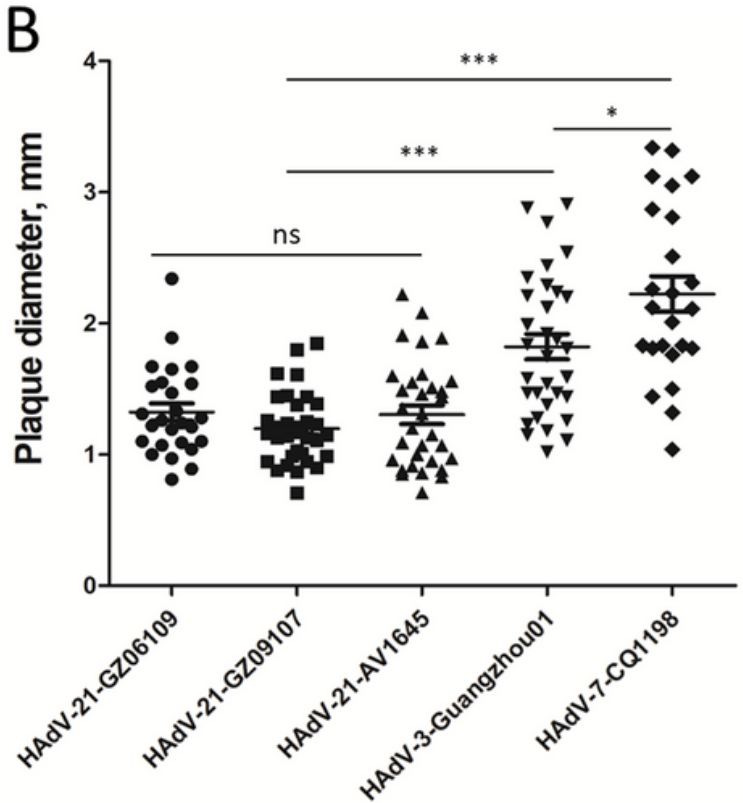

Figure 5 
Plaque formation and size distribution of the HAdV-21 isolates and references HAdV-21-AV-1645, HAdV-3Guangzhou01, and HAdV-7-CQ1198 (A) Plaque formation. (B) Size distribution. Plaque plates were incubated and stained with crystal violet for a total of 13 days in 6-well culture plates. ns, not significant; $*, p<0.05 ; * \star *, p<0.001$.

\section{Supplementary Files}

This is a list of supplementary files associated with this preprint. Click to download.

- TableS1.docx 